\title{
Mentalización en Psicoterapia: Discusión Sobre lo Explícito e Implícito de la Relación Terapéutica
}

\section{Mentalizing in Psychotherapy: Discussion on Explicit and Implicit of the Therapeutic Relationship}

\author{
Claudio Martínez \\ Universidad Diego Portales, Chile
}

(Rec: 15 de enero de 2009; Acept: 10 de abril de 2010)

\begin{abstract}
Resumen
La capacidad de mentalización es un logro del desarrollo y su cualidad está influida por las relaciones tempranas con las figuras de apego. La mentalización juega un rol central en la psicoterapia tanto como actividad mental de terapeuta y paciente como también en la tarea de promover esta capacidad en pacientes carentes de ella. En este trabajo se describe la utilidad de la mentalización para la psicoterapia, enfatizando la actividad del terapeuta al generar mentalización en el paciente, pero se discuten los mecanismos de integración entre lo explícito e implícito de este proceso. Se propone que mientras la mentalización explícita puede variar según el entrenamiento y formación del terapeuta, la mentalización implícita depende mucho más de la amplitud de conciencia (mindfulness) del terapeuta sobre sus estados mentales y su habilidad en expresar y poner en juego estos estados mentales en la psicoterapia.

Palabras clave: Mentalización, relación terapéutica, psicoterapia
\end{abstract}

\begin{abstract}
The capacity for mentalizing is a developmental achievement and its quality is influenced by the early relationships with attachment figures. Mentalizing plays a central role in psychotherapy, both as a mental activity of therapist and patient, as well as in the task of promoting the development of this capacity in patients who lack it. In this work the usefulness of mentalizing for psychotherapy is described, emphasizing the activity of the therapist in the generation of mentalizing in the patient, but the mechanisms of integration between the explicit and implicit of this process are discussed. I propose that while explicit mentalizing can change according to therapist degree of training, implicit mentalizing depends much more on the therapist's amplitude of consciousness (mindfulness) about his mental states and his ability to express and bring these mental states into psychotherapy.
\end{abstract}

Key words: Mentalizing, therapy relationship, psychotherapy. 


\section{Introducción}

La capacidad de los seres humanos de interpretar y comprender las propias conductas y las de otros es una función cognitiva esencial para la convivencia e interacciones en un mundo social.

Siempre que un terapeuta piensa en los estados mentales del paciente que tiene enfrente para así chequear la respuesta verbal o no verbal de aquel acerca de una intervención recién hecha, o bien imagina las emociones que puede estar sintiendo su paciente antes de proferir alguna interpretación, está ejerciendo su capacidad de mentalización. Cuando el terapeuta le solicita al paciente que diga por qué cree que ha sonreído al momento de escuchar una determinada intervención, se está estimulando al paciente que ejercite su capacidad de mentalización. Por otro lado, cuando ante un comentario de un paciente, el terapeuta, de manera no conciente, mueve aprobatoriamente su cabeza al tiempo que sonríe levemente y levanta sus cejas, podemos pensar que ese terapeuta está "empatizando" con su paciente, respuesta emocional que es resultado de ejercitar la mentalización (Allen, 2003). En todas estas situaciones, habituales en cualquier psicoterapia, estamos poniendo en práctica una función cognitiva que es connatural a las relaciones sociales, pero que adquiere una importancia y una complejidad particular en el contexto psicoterapéutico.

El concepto de mentalización fue introducido en la literatura psicoanalítica algo tardíamente en el contexto de estudios sobre el desarrollo mental infantil, y de allí ha sido exportado al ámbito de la psicoterapia.

$\mathrm{Su}$ importancia en este ámbito hace referencia a los hallazgos que la investigación ha entregado en lo últimos años sobre la relación entre el desarrollo de la mentalización y procesos psicopatológicos específicos como los trastornos limítrofes de personalidad (TLP). En este campo, el mayor aporte ha sido identificar la calidad de las relaciones de apego como un mediador del desarrollo de la mentalización, donde las situaciones de mala calidad de la relación de apego temprano o eventos de violencia y abuso durante la infancia darían lugar a una pobreza en la capacidad mentalizadora (Fonagy \& Target, 1997; Fonagy, Target \& Gergely, 2000). Las investigaciones en esta línea han aportado en el diseño de propuestas de intervención terapéuticas que buscan, como foco central, el aumento o desarrollo de la capacidad mentalizadora en pacientes que carecen de ella, en particular pacientes de condición TLP (Bateman \& Fonagy, 1999, 2001, 2004; Fonagy \& Target, 2002). Estas propuestas, a pesar de tener un buen sustento empírico, acarrean ciertas dificultades o, al menos, proponen desafíos a los clínicos que desean ponerlas en práctica. Una de ellas en la división que se ha descrito entre la mentalización explícita e implícita y en la poca claridad de la literatura acerca de la relación entre ambas. Una revisión de estas conceptualizaciones y de la vinculación entre ellas constituye el propósito central de este trabajo.
Primariamente se hace una síntesis de los aportes más relevantes en el tema de la mentalización, tanto en su vinculación con el desarrollo psicopatológico de esta capacidad como con la propuesta específica al ámbito terapéutico. Posteriormente se revisa la relación entre la mencionada división-explícita e implícita- de la capacidad mentalizadora, para finalmente proponer una integración concentrando la discusión sobre el acto psicoterapéutico.

\section{Mentalización, apego y psicopatología}

Mentalizamos cuando tratamos a otros como personas y no como objetos. Es un proceso en que le otorgamos sentido a nuestros estados mentales y a los de los otros. Aunque somos propensos a asociar la mentalización con compasión, hay que tener claro que nuestra mentalización no siempre significa benevolencia (Allen, 2003). Cuando no mentalizamos podemos tratar a los otros de las más degradantes maneras, por ejemplo podemos ser explotadores, utilizando a los otros solamente para conseguir nuestros fines. En los trastornos antisociales o psicopatía, por ejemplo, la capacidad de mentalización está al servicio de la manipulación y el engaño, en los sádicos estará para humillar y maltratar a otros, etc. Por el contrario, en la psicoterapia esta capacidad podrá ser usada para entender al paciente y empatizar con él. Por tanto, una cosa es el desarrollo o no de esta capacidad cognitiva y otra es su pragmática, su uso en las interacciones sociales.

La mentalización supone interpretar la conducta de uno mismo y de otros en términos de estados mentales intencionales, tales como deseos, sentimientos y creencias. Los estados mentales intencionales son acerca de algo. La intencionalidad divide marcadamente los seres mentales de los objetos físicos (Allen, 2003; Gomez \& Nuñez, 1998). Un mueble no puede ser "acerca" de algo, tan sólo es. Una mente, en cambio, se relaciona activamente con el mundo fuera de sí misma; una mente puede pensar acerca de un mueble, acerca de otra mente o acerca de sí misma. La intencionalidad de la mente está ligada a la capacidad representacional. Las representaciones mentales, como las creencias representan algo como siendo de cierta manera (Perner, 1994). Vemos una expresión facial como siendo alegre o enojada. A través de las representaciones nos independizamos de la realidad externa y, por ejemplo, podemos jugar. Para un niño un bloque de madera puede ser un camión y para un psicoanalista una botella de vino puede ser un pecho materno.

La capacidad de mentalización es un logro del desarrollo que se da en el contexto de un vínculo afectivo seguro. Las investigaciones iníciales de Fonagy y colaboradores mostraron que madres, evaluadas antes del nacimiento de sus hijos, con un apego seguro, eran más capaces de generar un ambiente para sus bebés que, a su vez, permitía tener bebés con un apego seguro (Fonagy, Steele, Moran, Steele, \& Higgitt, 1991). Este hallazgo inicial les permitió 
reformular las concepciones sobre la seguridad del apego y plantearon que cualquier noción de procesos internos inherentes a la seguridad del apego debe incluir la capacidad cognitiva de las madres o cuidadores de poder pensar acerca de sentimientos y su relación con las conductas de su bebé (Fonagy, Gergely, Jurist \& Target, 2002). Estos mecanismos cognitivos para procesar la experiencia intersubjetiva e interpersonal fueron ubicados dentro del amplio concepto de mentalización y ligados a las conocidas nociones de teoría de la mente y su papel en el desarrollo mental del infante (Premack \& Woodruff, 1978; Stern, 1985), pero llamando "función reflexiva" a la operacionalización de la capacidad de mentalización en el ámbito del apego.

Fonagy y sus colegas sugieren que la capacidad de la madre para contener en su mente complejos estados mentales, le permite, a su vez, mantener en mente la experiencia afectiva y emocional de su bebé, para así comprender el comportamiento de su hijo a la luz de estados mentales como emociones, sentimientos e intenciones (Fonagy \& Target, 1997; Fonagy, et al., 2002). Entregándole significado a su experiencia afectiva y re-presentándole esta experiencia al bebé de una manera regulada y metabolizada, la madre o cuidador establece las bases para un desarrollo de un sentido de seguridad, autenticidad y coherencia en el self del niño (Slade, Grienenberger, Bernbach, Levy \& Locker, 2005). En síntesis, la línea de investigación de Fonagy y sus colaboradores propone que el apego estimula las capacidades para la representación mental. El desarrollo cognitivo sería un producto de un complejo proceso psicológico que emerge en la proximidad a otro ser humano en la temprana infancia, pero la calidad del desarrollo de esta capacidad también dependerá de la calidad de esa temprana relación. Por ende, en ausencia de vínculos afectivos seguros, en circunstancias en que las llamadas figuras de apego no logran ejercer tareas que estimulen la capacidad mentalizadora en los niños, como actividades de atención conjunta o de mantener con el bebé un estado de sintonía emocional, los niños tendrán dificultad para discernir sus propios estados mentales o los estados mentales de los demás o, lo que es peor, a no diferenciar los estados internos de los ajenos (Allen, 2001; Fonagy, 2003).

Una forma de entender lo que los psicoanalistas llaman "patología pre-edípica o los teóricos del apego "inseguridad" o "desorganización", es considerar estas manifestaciones como una falla para desarrollar una rudimentaria capacidad para entrar completamente en la experiencia subjetiva propia y de los otros con confianza y no sobre defensas primitivas y distorsiones (Slade, 2005). Las raíces de esta inhabilidad pueden vincularse a las vulnerabilidades de la temprana infancia. Fonagy et al., (2002) describen varias fallas en la tarea materna de "espejamiento" (mirroring) de los estados emocionales del bebé. En algunos casos el "espejamiento" puede ser demasiado exacto o demasiado real, como por ejemplo una madre que responde al miedo de su hijo con su propio miedo, más que con una representación del miedo del bebé. En otros casos, puede que los padres marquen el afecto del infante, pero en una forma no contingente, creando así una falsa representación de la emoción del niño. Por ejemplo, una madre interpreta los llantos de su bebé como manipulaciones y decide responder ignorándolo hasta que el bebé se calle o su llanto exceda el tiempo o el volumen que la madre se autoinpone como límite para acudir y acogerlo. En tales situaciones, las representaciones del bebé de sus propios estados emocionales se distorsionan y el niño queda con un equivocada representación de sus estado emocional, por ejemplo debo llorar más tiempo del necesario, o debo callarme aunque me duela, o debo esperar que mi madre desee asistirme y no cuando yo lo necesito. Esto es lo que Winnicott (1971) llamó "falso self": una adaptación a los afectos y mentes de los otros que deja al self sintiéndose vacío y poco real, y al otro como un ser extraño que se internaliza, "self alien" dirá Fonagy (Bateman \& Fonagy, 2004), desconectado de la experiencia verdadera del self.

Tales cuidadores no reflexivos y con fallas en la regulación afectan el desarrollo del self del infante de manera muy profunda, particularmente cuando estas fallas de sintonía o insensibilidad son crónicas y el niño es forzado a internalizar los estados mentales distorsionados como si fueran una parte de sí mismos. Padres abusivos inundan la experiencia emocional de sus hijos con su propia rabia, miedo y angustia. El niño (y sus estados mentales) no es visto como él mismo, sino como una proyección y distorsión de los padres. El niño muchas veces toma el odio y agresión de los padres como propias, como una primitiva forma de identificarse con el agresor. Estas y otras adaptaciones, por patológicas que sean, son una forma de sobrevivir emocionalmente, son adaptaciones que sostienen, al mismo tiempo, estas relaciones con sus seres amados y, más tarde serán parte de sus relaciones adultas (Slade, 2000).

Una serie de investigaciones de Fonagy y sus colaboradores (Fonagy, et al., 2002; Bateman \& Fonagy, 2004) han arrojado cifras que cuantificaban la relación entre mentalización, situaciones traumáticas o abuso en la infancia y patología de la personalidad. Por ejemplo, encontraron que el $97 \%$ de los individuos que habían sufrido malos tratos y que tenían una baja capacidad de mentalización cumplía con los criterios diagnósticos de trastorno límite de la personalidad. En cambio sólo un $17 \%$ de los sujetos que había padecido abuso y maltrato y pertenecían al grupo de capacidad reflexiva alta, satisfacía esos mismos criterios.

Los individuos con una buena capacidad de mentalización logran comprender los pensamientos de sus padres y procesar adecuadamente los acontecimientos que les sirve para procesar la experiencia traumática. En el fondo, logran imaginar otras perspectivas para poder comprender las conductas de otras personas y no considerar sólo su experiencia subjetiva como la única realidad posible (Gabbard, 
2005). Los pacientes con TLP tienen, a menudo, la gran dificultad para abandonar un modelo de funcionamiento mental de "equivalencia psíquica" y poder funcionar con un modelo del tipo "como si", lo que les dificulta pensar en visiones diferentes acerca de la realidad percibida por ellos. Se aferran a su percepción particular de las cosas, considerando que lo que piensan es porque efectivamente sucede ha sucedido en la realidad y los otros deberían percibir y pensar lo mismo. Esta dificultad condiciona su capacidad de reconocer la transferencia con el terapeuta como una experiencia "como si", lo que muchas veces hace que estén convencidos que su interpretación de la conducta del terapeuta, de sus expresiones faciales o de sus comentarios, es la única posible y la "correcta" y no una de tantas dentro de muchas otras posibles.

Por tanto, una de las principales y más difíciles tareas para terapeutas que tratan a personas con TLP es generar en ellos un "modelo" de funcionamiento mental que les permita diferenciar entre sus percepciones de la realidad y la de los demás. Considerando que su forma de pensar las conductas de otros son representaciones o interpretaciones que están basadas en sus creencias internas, sus sentimientos y sus experiencias pasadas.

\section{Mentalización en Psicoterapia}

Para abordar el tema de la mentalización en psicoterapia es necesario separar la mentalización como una función cognitiva utilizada de modo habitual por los terapeutas en el ejercicio del acto psicoterapéutico, de la especificidad de un modelo de tratamiento que tiene como foco central la mentalización, ya sea como parte fundamental de la técnica del terapeuta o como meta del tratamiento de pacientes que carecen de esta capacidad. Como el propósito de este trabajo apunta más en la dirección de analizar la mentalización como parte de todo acto psicoterapéutico, dejaremos este aspecto para después y comenzaremos con una breve descripción de un tratamiento específico para pacientes que padecen TLP y que tiene como eje central la mentalización.

\section{Tratamiento basado en la mentalización (TBM)}

Como se dijo más arriba, las personas diagnosticadas con TLP, en su mayoría, tendrían una pobre o nula capacidad de mentalización (Fonagy et al., 2002), lo que contribuye a funcionamiento psicológico e interpersonal problemático, distorsiones en su capacidad de interpretar la realidad, severas dificultades en la regulación de sus emociones y un sentido de sí (self) que se caracteriza por sentimientos de vacío y de inautenticidad. Bateman \& Fonagy (2004) han propuesto un tratamiento específico para tratar esta patología basándose en las investigaciones de Fonagy y sus colegas (Fonagy, Target, \& Gergely, 2000) sobre la relación entre el apego y el desarrollo normal o patológico de la capacidad de mentalización. En el corazón de su propuesta está la creencia, que comparto, de que la psicoterapia tiene el potencial de recrear una matriz interaccional de apego en la cual la mentalización puede desarrollarse, puesto que el mayor valor de la psicoterapia es la experiencia de tener al frente a otro ser humano conectado "mente a mente" (Fonagy \& Bateman, 2006, p.415). Teniendo en cuenta esto como telón de fondo, los elementos claves de este tratamiento pueden resumirse en lo siguiente:

1. El terapeuta debe focalizarse en sus intervenciones en el estado mental actual del paciente, en su "aquí y ahora" mental (pensamientos, sentimientos, deseos y necesidades), con el propósito de estimular la construcción conjunta de representaciones de estos estados internos. Este aspecto constituye una habilidad del terapeuta de estar constantemente preguntando y preguntándose sobre los estados internos y cómo estos estados internos puede explicar lo que está sucediendo "aquí y ahora" entre ambos (Bateman \& Fonagy, 2004).

2. El terapeuta evita situaciones en que el paciente hable de estados mentales que no puedan ligarse a una realidad subjetivamente sentida. Esta es una variante de los tratamientos psicoanalíticos tradicionales, pues se evita hablar de contenidos inconscientes, de focalizar en el pasado o de buscar el insight. Más bien, se persigue recuperar la capacidad de mentalización, buscando la coherencia de las representaciones y la integración de los estados intencionales.

3. La terapia, sea en modalidad grupal o individual, estimula la creación de un área transicional de relaciones en la cuál pensamientos y emociones puedan desplegarse e integrarse (Fonagy \& Bateman, 2006). Este espacio de transición permite establecer puentes entre la experiencia afectiva primaria y su representación simbólica. En la terapia se intenta fortalecer permanentemente el sistema representacional secundario.

4. El fenómeno transferencial no es utilizado en este tratamiento como una simple repetición del pasado o un desplazamiento. La transferencia es experimentada por los pacientes con TLP como algo real, preciso y actual y esto requiere ser aceptado por el terapeuta o por el equipo tratante.

5. Mantener al paciente en mente es la forma en que el terapeuta puede, de mejor manera, proveer un reflejo empático de las emociones y sentimientos que se despliegan en el tratamiento. La tarea del terapeuta es ser lo más exacto posible en representar el estado afectivo del paciente y para ello debe sintonizar con el paciente desde su propio estado emocional (Bateman \& Fonagy, 2004).

6. Las inevitables actuaciones de los pacientes a través del curso del tratamiento no son interpretadas o entendidas en términos de su significado inconsciente, 
sino en términos de la situación y afectos presentes inmediatamente antes que la actuación sucediera.

Las estrategias descritas aquí y el uso de la transferencia forman el núcleo de la TBM. El modelo reflexivo o de mentalización estimula más a pensar en las conductas que en cambiarlas, aunque habitualmente los cambios en el comportamiento siguen a cambios en el pensamiento y en las representaciones. En el fondo la propuesta de una psicoterapia mentalizadora es ayudar a los pacientes a aprender a leer intenciones y estados mentales, sin distorsionarlos (Slade et al., 2005). Este propósito queda más que claro con un tratamiento diseñado particularmente para pacientes que poseen especial dificultad en estas capacidades, pero, tal como se mencionó previamente, qué ocurre en cualquier tratamiento terapéutico, con cualquier otro tipo de paciente. ¿El propósito del tratamiento no es más o menos el mismo? ¿No buscamos siempre que nuestros pacientes desarrollen su capacidad de pensar en sí mismos, en sus estados internos y en las motivaciones e intenciones de los demás? Mentalizando podemos imaginar variadas formas en que otros pueden estar pensando o sintiendo, preguntándonos por qué ellos hacen lo que hacen, procurando hacer sus acciones inteligibles. Gran parte de lo que hacemos en psicoterapia es estimular esta capacidad de meta-representar de los pacientes para que reflexionen acerca de sí mismos, sus sentimientos y acciones, considerando diferentes perspectivas (Allen, 2003). En psicoterapia intentamos ayudar a nuestros pacientes a ser curiosos acerca de cómo sus mentes trabajan y cómo son las mentes de los demás. Nos comprometemos con ellos en un proceso de exploración de varios posibles significados de sus sentimientos, sueños y acciones. Por tanto, debemos concentrarnos en la mentalización como una capacidad o función presente en el proceso psicoterapéutico como un todo y no sólo como una meta de un tratamiento específico.

\section{Mentalización explícita e implícita en la psicoterapia}

Con el propósito de profundizar en el rol que la mentalización puede jugar en todo proceso psicoterapéutico es importante distinguir entre los niveles explícitos e implícitos de la capacidad de mentalización (Perner, 1994). En pocas palabras, mentalizamos explícitamente cuando pensamos reflexivamente acerca de las acciones de los otros y de nosotros mismos. Es un proceso relativamente consciente, reflexivo, deliberado y no inmediato. Por el contrario, mentalizamos implícitamente cuando interactuamos intuitiva e irreflexivamente. Es un proceso inconsciente, procedural e inmediato (Allen \& Fonagy, 2002).

Como psicoterapeutas, habitualmente nos focalizamos en explicar y otorgar significados a los estados mentales del paciente. Esta mentalización explícita tiene la ventaja de ser una reflexión consciente, de generar un monitoreo explícito en la interacción. El lenguaje es el medio representacional por excelencia en este tipo de mentalización, pues es la palabra la que capitaliza este nivel de representación y permite, entre otras cosas, adoptar múltiples perspectivas mentales sobre una misma realidad. Además, el lenguaje permite que esta forma de conocimiento de las mentes -propia y de otros- sea sistematizada y decante en una narrativa coherente de la propia autobiografía y, por cierto, del sentido del propio self. Gran parte de nuestro trabajo psicoterapéutico encierra este propósito y se sirve de lo explícito de la mentalización en esta tarea.

Sin embargo, mentalizar explícitamente no suplanta la mentalización implícita. Nosotros apenas somos conscientes de nuestra mentalización implícita la mayor parte del tiempo, por más que intentemos explicar lo implícito, por ejemplo cuando tratamos que el paciente ponga atención sobre el significado de su puño apretado. Y mentalizamos implícitamente siempre que estamos comprometidos emocionalmente en interacciones con otros. Podría decirse que la distinción entre explícito e implícito es un asunto de grado, pero la distinción es válida, pues establece la desigualdad entre mentalización y la actividad reflexiva consciente. En el fondo, entre lo explícito e implícito hay algo más que un continuo, al menos en lo que a la psicoterapia se refiere. Aunque habitualmente nuestra atención terapéutica está volcada hacia la explicación, el acto psicoterapéutico está fundado sobre la mentalización implícita. Es a través de este proceso implícito que el terapeuta compromete al paciente en una potencial relación terapéutica. Cuando un paciente después de una primera entrevista declara que seguirá viniendo porque "creo que usted me entiende". El terapeuta, que aún no ha explicitado ninguna opinión reflexiva acerca de lo que entiende o no del paciente, se puede preguntar: “¿cómo puedo haber entendido sin entender?”. Es probable que en esta experiencia común esté actuando la mentalización implícita en la forma de comunicación no verbal y, lo más importante, no consciente para el terapeuta. En general, cualquiera sea el contenido del diálogo, gran parte de la mentalización implícita es no verbal. Las expresiones faciales, la prosodia del lenguaje, los gestos y posturas, son todas expresiones de lo que Allen \& Fonagy (2002) llaman "responsividad sensible" en un contexto de apego y sintonía o empatía en un contexto terapéutico (p.29). Para estos autores, y en general para una buena parte de la literatura en esta línea, la relación entre lo implícito y explícito en la psicoterapia es una relación jerárquica, donde la mentalización implícita es una especie de telón de fondo donde se desplegará la mentalización explícita. Eventualmente la explicación terapéutica servirá para iluminar lo implícito del proceso terapéutico. Lo óptimo sería que el discurso terapéutico pudiera integrar la mentalización implícita y explícita en un proceso de redescripción representacional, explicando lo implícito, creando conciencia de sutiles combinaciones de afectos, atendiendo a contradicciones entre deseos y 
emociones (Linehan, 1993; Linehan, Armstrong, Suarez, Allmon \& Heard, 1991). El conocimiento inarticulado de la experiencia vivida nos permite darle sentido a las ideas y teorías, el conocimiento pre-verbal e implícito sería la raíz del conocimiento y de la razón explícita (Toulmin, 2001, citado en Allen \& Fonagy, 2002). Dicho de esta manera, es difícil estar en desacuerdo y debemos avalar la meta integradora que se propone. Incluso, desde una perspectiva psicodinámica, la meta de la psicoterapia no sería alcanzar específicos insights, sino fortalecer la capacidad para el insight en un sentido amplio, esto es, haciendo sentido de los otros y de sí mismo, implícita y explícitamente (Allen \& Fonagy, 2002). ¿Pero cómo un terapeuta hace esto? ¿Cómo se integran, en medio del proceso y en un momento cualquiera de la psicoterapia, lo explícito e implícito de la mentalización, sea esta del paciente o del terapeuta?

\section{Discusión}

Como psicoterapeutas debemos mentalizar para generar mentalización en nuestros pacientes. Es a través de nuestra propia mentalización que comprometemos a nuestros pacientes en el proceso de mentalizar y, a su vez, a través de su mentalización se comprometan en nuestro proceso. Llevamos a la psicoterapia nuestras propias competencias mentalizadoras, nuestra propia sensación de seguridad, nuestra historia de apego y la actual representación que tenemos de ella. En suma, colocamos en este proceso todos aquellos elementos que contribuyen o entorpecen un buen desarrollo de mentalización. Ese es el material con el que llegamos a trabajar con nuestros pacientes. Nos ayuda la experiencia y el entrenamiento, el dominio de nuestras teorías y todo el conocimiento teórico del que disponemos. Elementos que, sobre todo, serán fundamentales para la mentalización explícita.

También debemos estar conscientes de nuestros temores, inseguridades y habituales formas de responder ante la amenaza. Cuando un paciente se paraliza en su proceso de mentalizar al conectarse, por ejemplo, con elementos traumáticos de su historia, también nosotros podemos sentirnos amenazados e inseguros. Sólo la confianza mutua permitirá salir de situaciones de esta naturaleza. El proceso de explorar el mundo mental requiere de un sentido de seguridad y confianza de ambas partes. Como dice Allen (2003), "se necesitan dos para mentalizar terapéuticamente" (p. 105).

Es posible que la mentalización explícita por sí sola nos lleve a intentar "objetivar" a nuestro paciente o lo que a él le ocurre, algo así como un acto reflexivo desapegado o desvinculado. Quedarse exclusivamente en este nivel, creo, irremediablemente nos llevaría al fracaso. Sugiero, en cambio, que la conexión que realicemos durante el proceso de psicoterapia con nuestra capacidad de mentalizar implícitamente, deberá comandar el trabajo terapéutico, constituyéndola en nuestra principal fuente de información.

Esta conexión ocurriría de dos maneras. Una de ellas es que el proceso implícito proporcione información que podamos luego poner al servicio de la mentalización explícita. Esta vía es concordante con las propuestas de Allen \& Fonagy (2002) y la literatura revisada sobre el tema, pero no vincula claramente los dos niveles de mentalización. Por el contrario, aparecen como procesos separados (ver figura 1) que ocurrirían en momentos temporalmente distintos.

FIGURA 1.

Modelo basado en Allen \& Fonagy

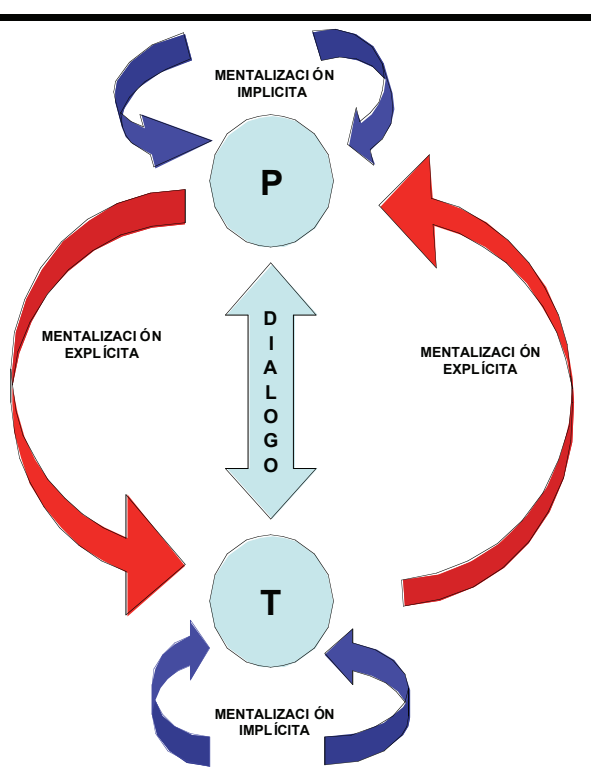


La otra vía es, a mi juicio, mucho más importante: La mentalización implícita aporta directamente a la sintonía afectiva entre paciente y terapeuta. Y a su vez, sólo esta sintonía mutua permite la coherencia e integración entre los niveles implícito y explícito de mentalización. De otra manera, es posible que aparezcan descoordinaciones entre estos niveles y algunas intervenciones del terapeuta resulten incoherentes, atemporales y diacrónicas. Dicho de otro modo, la sintonía mutua es más que un buen fondo, de buen vínculo y buena voluntad, sugiero que es el único canal válido para que la coherencia entre lo explícito e implícito de la mentalización funcione. Por tanto, la relación entre la mentalización implícita y la sintonía es de una comunicación de dos vías, en ambos sentidos y de una mutua necesidad que, la mayor parte de las veces, requiere de regulaciones entre ambos participantes. Aquí se vislumbra el papel principal de la mentalización explícita: Como reguladora de esta relación entre sintonía afectiva y mecanismos implícitos. Es probable, y lo digo a modo de hipótesis, que otros mecanismos reguladores de paciente y terapeuta jueguen aquí un importante papel en la fluidez de comunicación entre la mentalización y la sintonía. Pero, estimo, que la explicitación de los procesos mentalizadores es una herramienta que está mucho más a la mano para los terapeutas. Por ejemplo, para que dos individuos puedan sintonizar es necesario que previamente se acerquen mutuamente con la suficiente apertura y confianza para que ninguno adopte una postura rechazadora o desconectada. Si ambos, o uno de ellos, está en una postura de rechazo y desconexión es imposible que haya sintonía. Aquí es donde puede funcionar la mentalización explícita del terapeuta, o de ambos, para regular esta interacción al verbalizar las emociones que están siendo actualizadas o explicitar metacomunicativamente la experiencia afectiva en acción. De esa manera puede aumentar la confianza, disminuyen las barreras defensivas, aumenta la superficie de contacto y es posible sintonizar afectivamente. A su vez, esta sintonía facilita la mentalización implícita que consecuentemente aportará mayor confianza o nuevos conocimientos que pueden ser mentalizados explícitamente (ver figura 2).

FIGURA 2.

Modelo Propuesto

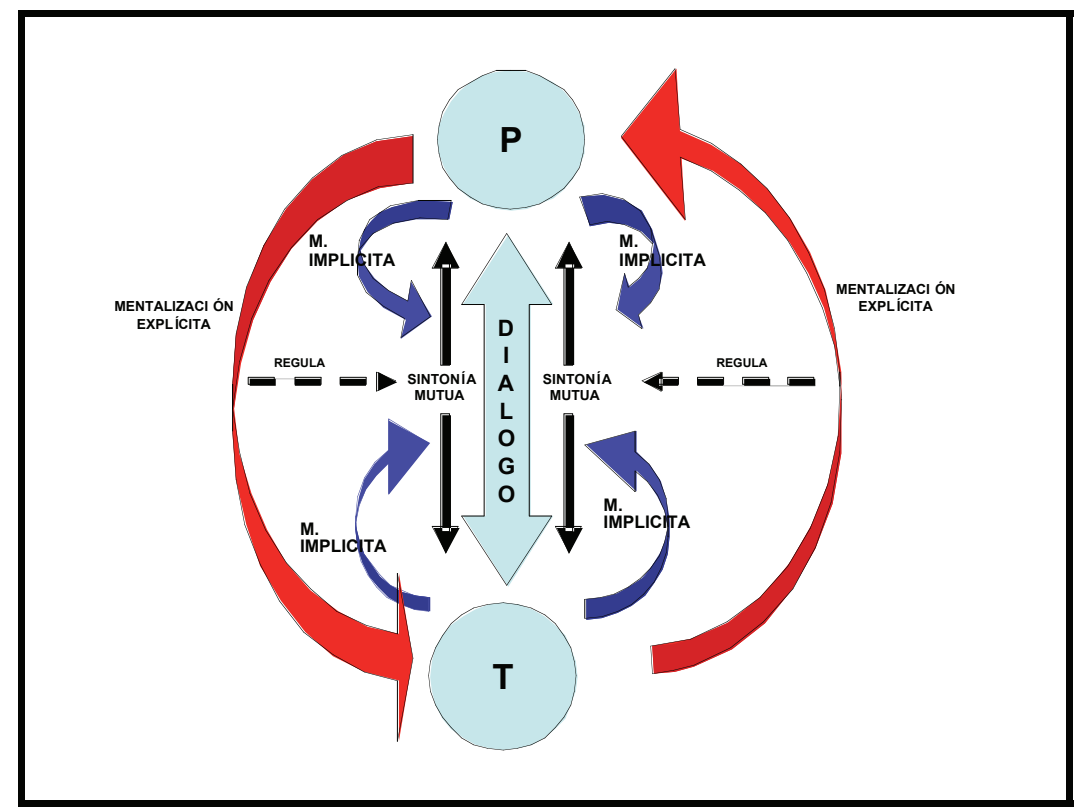

Es como un "loop" de conexiones entre los niveles de mentalización, pero que pasa por la capacidad del terapeuta de acceder al nivel más implícito de la vivencia. En el fondo, es la capacidad del terapeuta para acceder a su intuición, abriendo un camino hacia esa área menos consciente y más automática (Myers, 2002).

¿Es esto posible? ¿Cómo podemos acceder a un área que, por definición está fuera de nuestra capacidad consciente? Quizás la respuesta pase por la noción de inconsciente $y$, al menos por ahora, la consideración que no hay un "área" inconsciente, sino procesos inconscientes o más bien, menos accesible a la conciencia. Sin pretender discutir filosóficamente el punto, diremos aquí que la accesibilidad de la conciencia puede ser sólo un tema de "intención", de "atención" y de "actitud". Remarco estos tres aspectos porque forman parte de lo que se ha llamado los "axiomas de la mindfulness" o "amplitud de conciencia" (Shapiro, Carlson, Astin \& Freedman, 2006). En breve, 
sugiero que un terapeuta que se propone atender, durante el mayor tiempo posible, a la experiencia interna y externa del momento a momento del proceso terapéutico, debe hacerlo en una actitud contenedora (segurizante), no interpretativa, no evaluadora o prejuzgadora, para alcanzar la amplitud de conciencia necesaria y poder acceder a aquellos propios procesos habitualmente intuitivos o menos conscientes, y sobretodo, a los procesos menos conscientes del paciente.

Por tanto, la integración entre los niveles explícito e implícito de la mentalización pasa, por una parte, por un aspecto intersubjetivo entre terapeuta y paciente de sintonía y regulación afectiva mutua, y por otra, de una capacidad del terapeuta de ampliar su conciencia a su experiencia interna para acceder a la información que su cuerpo y su intención pueden darle y así, llevar este conocimiento de si mismo al conocimiento explícito del otro. En otras palabras, es como integrar permanentemente lo simétrico y asimétrico de la relación terapéutica.

Hemos visto en este trabajo que la capacidad de mentalización es una función cognitiva que parece ser fundamental en la comunicación y relaciones afectivas entre seres humanos. Aún más, el adecuado desarrollo y específicas cualidades parecen depender estrechamente de la calidad de las relaciones tempranas. En esta área, los trabajos de Fonagy y sus colegas, han demostrado que existen relaciones muy directas entre calidad del apego, mentalización y patología de la personalidad. Esto ha significado generar modelos de tratamiento específicos para TLP teniendo como foco central el desarrollo y estimulación de la mentalización.

Se ha enfatizado aquí que la mentalización en la psicoterapia es un elemento central del trabajo terapéutico, ya sea como meta del tratamiento para aquellos individuos que tienen déficit en esta capacidad cognitiva, y también como parte esencial de la labor de todo terapeuta. En este último plano, la investigación empírica ha mostrado que un proceso psicoterapéutico funciona más completo y eficiente si los niveles explícitos, ligados al lenguaje, e implícitos, ligados a los procesos no verbales e intuitivos, de la mentalización se integran. En la literatura donde ello se afirma no se establece con claridad los mecanismos para lograr tal integración. En este trabajo se ha discutido una posible integración entre los niveles explícito e implícito de la mentalización. Un modelo que supone dos vías o niveles interdependientes. Una vía, de carácter intersubjetivo y, en ese sentido más simétrica, supone que la mentalización implícita del terapeuta y del paciente ayudan a la sintonía afectiva entre ambos, lo que junto a otros procesos de regulación mutua (ej. representaciones de apego) permiten, a su vez, aumentar la mentalización implícita. Este nivel está estrechamente ligado a la segunda vía, más asimétrica, pues depende fundamentalmente de las aptitudes del terapeuta. En este sentido, se sugiere que la amplitud de conciencia (mindfulness) del terapeuta le permitiría acceder a los niveles más implícitos de mentalización y en un acto intencional y ligado a su tarea, llevar esta información de sí mismo y de su paciente a un nivel explícito de mentalización. Aquí, la mentalización explícita se convierte en un proceso regulador más y aporta a la sintonía afectiva con el paciente. Por tanto, ambos niveles pueden interactuar, aportando información al proceso terapéutico. Sin embargo, se enfatiza que todo el proceso debiera ser comandado por este nivel implícito, no en el sentido de su importancia relativa, sino sugiriendo que acceder a través de esa vía puede ser más eficiente. Esta afirmación se basa en la importancia que en los últimos años se ha dado al tema de la regulación afectiva en la díada terapeuta-paciente ( ver Beebe \& Lachman, 1998, 2002; Fosha, 2001; Lyons-Ruth, 1999; Martínez 2010; Martínez, Tomicic, Medina, \& Krause, 2011; Tomicic Martínez, Altimir, Bauer, \& Reinoso, 2009), con sustento en la regulación mutua entre madre e hijo por un lado (Beebe \& Lachman, 1988), y en estudios neurobiológicos sobre la coordinación de las estrategias afectivas del self (Schore, 1994). También aquí caben aquellos estudios que enfatizan la comunicación no verbal o de aspectos expresivos - como tono de voz o configuración facial - en la relación terapéutica y la potencialidad que la experiencia a este nivel tendría en el desarrollo de la subjetividad (Español, 2007; Stern, 2000; Trevarthen, 2008).

Por otro lado, el tema de la amplitud de conciencia se relaciona con otros conceptos, como el de "autoconciencia o "self-awareness" que, entre otros, pertenece a los factores de la llamada "inteligencia emocional" (Barbudo \& Burbach, 2006), pero que últimamente ha sido investigado como una tarea del terapeuta y relacionado con una evaluación más positiva del paciente acerca del proceso terapéutico (Williams \& Fauth, 2005).

Por último, tanto el tema de la sintonía afectiva o regulaciones diádicas entre terapeuta y paciente, como la capacidad de "autoconciencia" del terapeuta, que aquí hemos enfatizado como mecanismo para aumentar la conexión entre los niveles explícitos e implícitos de mentalización, son aspectos que requieren mucha más investigación empírica que puede contribuir al entendimiento más fino de la importancia de la relación terapéutica. Creo que este puede ser un camino que amplíe y profundice en el concepto de alianza terapéutica, fenómeno clínico que se ha estudiado ampliamente en los últimos 50 años y que forma parte de los llamados "factores comunes" de la psicoterapia y que explica gran parte del cambio psicológico de los pacientes en el tratamiento. A pesar de la multiplicidad de investigación en el tema, el concepto alianza sigue siendo algo vago, particularmente en su componente vincular que aún no hace evidente de qué manera se convierte en un ingrediente activo tan potente para el cambio psicoterapéutico. Quizás, la profundización en todos los aspectos implícitos y menos reflexivos de la interacción permita entender un fenómeno que continúa generando preguntas a los investigadores. 


\section{Referencias}

Allen, J.G. (2001). Traumatic relationships and serious mental disorders. Chichester, UK: Wiley.

Allen, J. G. (2003). Mentalizing. Bulletin of the Menninger Clinic, 67, 2, 91-112.

Allen, J.G., \& Fonagy, P. (2002). The development of mentalizing and its role in psychopathology and psychotherapy. Topeka, KS: The Menninger Clinic, Research Department.

Barbudo, J. E., \& Burbach, M.E. (2006).The emotional intelligence of transformational leaders: A field study of elected officials. Journal of Social Psychology, 146, 1, 51-64.

Bateman, A., \& Fonagy, F. (1999).The effectiveness of partial hospitalization in the treatment of borderline personality disorder - a randomized controlled trial. American Journal of Psychiatry, 156, 1563-1569.

Bateman, A., \& Fonagy, F. (2001). Treatment of borderline personality disorder with psychoanalytically oriented partial hospitalization: An 18- month follow-up. American Journal of Psychiatry, 158, 1, 36-42.

Bateman, A., \& Fonagy, P. (2004). Psychotherapy for borderline personality disorder: Mentalization based treatment. Oxford: Oxford University Press.

Beebe, B., \& Lachmann, F.M. (1988). The contribution of mother-infant mutual influence to the origins of self and object representations. Psychoanalytic Psychology, 5, 305-337.

Beebe, B., \& Lachmann, F. (1998). Co-constructing inner and relational processes: Self and mutual regulation in infant research and adult treatment. Psychoanalytic Psychology, 15, 1-37.

Beebe, B., \& Lachmann, F. (2002). Infant research and adult treatment: Co-constructing interactions. Hillsdale, NJ: Analytic Press.

Español, S. (2007). Experiencia estética y desarrollo humano. Las artes temporales en la génesis de procesos psicológicos complejos. Psykhe, $16,123-133$.

Fonagy, P. (2003). The development of psychopathology from infancy to adulthood: The mysterious unfolding of disturbance in time. Infant Mental Health Journal, 24, 212-239.

Fonagy, P., \& Bateman, A. (2006). Mechanisms of change in mentalization-based treatment of BPD. Journal of Clinical Psychology, 62, 411-430.

Fonagy, P., \& Target, M. (1997).Attachment and reflective function: Their role in self-organization. Development and Psychopathology, 9, 679-700.

Fonagy, P., \& Target, M. (2002). Early intervention and the development of self- regulation. Psycholanalytic Inquiry, 22, 307-335.

Fonagy, P., Target, M., \& Gergely, G. (2000).Attachment and borderline personality disorder: A theory and some evidence. Psychiatric Clinics of North America, 23, 103-122.

Fonagy, P., Gergely, G., Jurist, E., \& Target, M. (2002). Affect regulation, mentalization and the development of the self. New York: Other Press.

Fonagy, P., Steele, H., Moran, G., Steele, M., \& Higgitt, A. (1991).The capacity for understanding mental states: The reflective self in parent and child, and significance for security of attachment. Infant Mental Health Journal, 13, 200-217.

Fosha, D. (2001). The dyadic regulation of affect. Journal of Clinical Psychology, 57, 227-242.

Gabbard, G.O. (2005). Mind, brain and personality disorders. American Journal of Psychiatry, 162, 648-655.
Gomez, J.C., \& Nuñez, M. (1998). La mente social y la mente física: Desarrollo y dominios de conocimiento. Infancia y Aprendizaje, 84, 5-32.

Linehan, M.M. (1993). Cognitive-behavioural treatment of borderline personality disorder. New York: Guilford Press.

Linehan, M.M., Armstrong, H.E., Suarez, A., Allmon, D., \& Heard, H. (1991). Cognitive-behavioural treatment of chronically parasuicidal borderline patients. Archives of General Psychiatry, 48, 1060-1064.

Lyons-Ruth, K. (1999). The two-person unconscious: Intersubjective dialogue, enactive relational representation, and the emergence of new forms of relational organization. Psychoanalytic Inquiry, 19, 576-617.

Martínez, C. (2010). Análisis Dialógico de la Regulación Mutua en Psicoterapia. Tesis Doctoral, Escuela de Psicología, Pontificia Universidad Católica de Chile. Santiago, Chile.

Martínez, C., Tomicic, A., Medina, L., \& Krause, M. (2011) A Microanalytical Look at Mutual Regulation in Psychotherapeutic Dialogue: Dialogic Discourse Analysis (DDA) in Episodes of Rupture of the Alliance. En prensa.

Myers, D.G. (2002). Intuition: Its powers and perils. London: Yale University Press.

Perner, J. (1994).Comprender la mente representacional. Barcelona: Paidós.

Premack, D., \& Woodruff, G. (1978).Does the chimpanzee have a theory of mind? Behavioral and Brain, 1, 515-526.

Schore, A.N. (1994). Affect regulation and the origin of the self: The neurobiology of emotional development. Hillsdale, NJ: Erlbaum.

Shapiro, S.L., Carlson, L.E., Astin, J.A., \& Freedman, B. (2006). Mechanisms of mindfulness. Journal of Clinical Psychology, 62, 3, 373-386.

Slade, A. (2000). The development and organization of attachment: Implications for psychoanalysis. Journal of the American Psychoanalytic Association, 48, 1147-1174.

Slade, A., Grienenberger, J., Bernbach, E., Levy, D., \& Locker, A. (2005). Maternal reflective functioning, attachment, and the transmission gap: A preliminary study. Attachment and Human Development, 7, 3, 283-298.

Stern, D. (1985).El mundo interpersonal del infante. Buenos Aires: Paidós.

Stern, D. (2000). Putting time back into our considerations of infant experience: A microdiachronic view. Infant Mental Health Journal, 21, 21-28.

Tomicic, A., Martínez, C. Altimir, C., Bauer, S., \& Reinoso, A. (2009). Coordinación vocal como una dimensión de la regulación mutua en psicoterapia. Revista Argentina de Clínica Psicológica, XVIII, 1, 31-41.

Toulmin, S. (2001). Return to reason. Cambridge, MA: Cambridge University Press. En Allen, J.G., \& Fonagy, P. (2002). The development of mentalizing and its role in psychopathology and psychotherapy. Topeka, KS: The Menninger Clinic, Research Department.

Trevarthen, C. (2008). The musical art of infant conversation: Narrating in the time of sympathetic experience, without rational interpretation, before words. Musicae Scientiae, Special Issue, 2008, 11-37.

Williams, E.N., \& Fauth, J. (2005). A psychotherapy process study of therapist in session self-awareness. Psychotherapy Research, 15, 374-381. Winnicott, D. W. (1971). Realidad y juego. Barcelona: Gedisa. 